\title{
Color Image Edge Detection: A Survey
}

\author{
Smaranika Subhasini \\ Department of Electronics and Communication Engineering \\ NIT Nagaland, Dimapur, Nagaland, India \\ Kh.Manglem Singh \\ Department of Computer Science Engineering \\ NIT Manipur, Imphal, Manipur, India
}

\begin{abstract}
This paper presents a comprehensive review on various color edge detection techniques. Several edge detection techniques have been proposed in literature to detect edges present in monochrome as well as color images. Some commonly used first derivative edge operators and second derivative edge operators such as Roberts, Sobel, Prewitt, Compass, Laplacian of Gaussian (LoG), Canny, Marr-Hildreth and Haralick are discussed here and then the techniques are further extended to vector-valued techniques. In the traditional color edge detection techniques, the color image is first separated into three different channels such as $R, G$ and $B$ before processing and then the algorithm is applied on the respective channels and in the end all channels are combined to produce the result. But in the Quaternion based techniques the color pixel is considered as a single unit as a result of which the geometrical transformations and three dimensional rotation are easier to perform on it compared to the RGB domain.
\end{abstract}

Keywords - color edge detection, monochromatic techniques, vector-valued techniques, Quaternions.

\section{INTRODUCTION}

In digital image processing, edge detection plays an important role in image analysis and computer vision areas. The high level image processing applications such as object recognition, object tracking, robot vision etc. depends upon the performance of edge detection technique. Edges may be defined as boundaries between distinct regions present in a particular image and they contain the most relevant information of the image. There may be different regions present in an image which are characterized by the properties like gray level, texture, intensity etc. In case of color image processing, Color images require more memory space for storage than grey scale images and also the transmission of color information requires a larger bandwidth. By using an efficient edge detection technique, the unnecessary details of a color image can be discarded and the useful information can be stored for further processing. This can effectively reduce the memory space for storing the color information and lower the transmission bandwidth.

The edge detection techniques can be broadly classified as:

(1) Edge detection in grey-scale images

(2) Edge detection in color images

The fundamental difference between a grey-scale image and a color image is, the pixel in a geay-scale image is a scalar valued function whereas in color image, a pixel is considered as a vector valued function as it consists of three color components (red, green and blue). Due to this, vector valued techniques are preferred for edge detection in color images.

\section{AN OVERVIEW OF SOME CLASSIC EDGE DETECTORS}

Many edge detection techniques have been proposed for grey-scale images in image processing literature. These edge detectors are categorized as:

(1) Edge detection methods based on first order derivative

(2) Edge detection methods based on second order derivative

These gradient based operators follow the basic three steps for detecting an edge in an image. 
Step 1. Noise Reduction: As the derivative based techniques are more sensitive to noise, so noise reduction is a very important step before edge detection

Step 2. Detection of Edge Pixels: The detectors respond strongly to abrupt changes or edges in an image

Step 3. Edge Localization: It helps in removing false edges and also repairs broken lines to give meaningful information contained in the image.

\section{(1) First derivative based Edge Detectors}

\section{A. Roberts Operator}

In 1965, Lawrence Roberts [1] proposed a cross gradient operator which uses a two dimensional mask for edge detection. This is the simplest form of edge detector used earlier for detection of edges in grey scale images

\begin{tabular}{|c|c|}
\hline 0 & -1 \\
\hline 1 & 0 \\
\hline
\end{tabular}

(a)

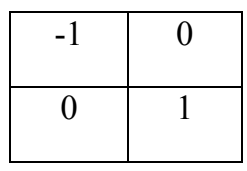

(b)

Figure 1: Robert's 2-D Kernels

\begin{tabular}{|l|l|l|}
\hline P1 & P2 & P3 \\
\hline P4 & P5 & P6 \\
\hline P7 & P8 & P9 \\
\hline
\end{tabular}

Figure 2: An image $\mathrm{f}(\mathrm{x}, \mathrm{y})$ of size $3 \times 3$

The algorithm of this operator can be summarized in the following three steps:

Step-1: Calculate the gradients ( $g_{\mathrm{x}}$ and $\mathrm{g}_{\mathrm{y}}$ ) of the image by applying first order derivatives

$$
\mathrm{g}_{\mathrm{x}}=\delta f / \delta x=(\mathrm{P} 2-\mathrm{P} 4) \quad \mathrm{g}_{\mathrm{y}}=\delta f / \delta y=(\mathrm{P} 1-\mathrm{P} 5)
$$

Step-2: Calculate the magnitude of the gradient by sum of the squares method

$$
|\mathrm{G}|=\sqrt{\left(g_{x}^{2}+g_{y}{ }^{2}\right)}
$$

Step-3: Convolve the gradients with the 2-D Roberts Kernel to detect edges in an image

$$
\begin{aligned}
& g_{x}=h_{x} * f(x, y) \\
& g_{y}=h_{y} * f(x, y)
\end{aligned}
$$

Where $\mathrm{h}_{\mathrm{x}}$ and $\mathrm{h}_{\mathrm{y}}$ are appropriate convolution masks (kernels).

The direction of the gradient can be calculated by the following relation:

$$
\theta=\tan ^{-1}\left(g_{\mathrm{y}} / \mathrm{g}_{\mathrm{x}}\right)
$$

\section{B. Sobel Operator}

Roberts mask is an even sized mask $(2 \times 2)$ which is unsuitable for implemention as the edge points after convolution operation correspond to ( $\mathrm{r}-1 / 2, \mathrm{c}-1 / 2)$. This can be avoided by using a $3 \times 3$ mask. 
The Sobel operator [2] uses a 2 -D mask of size $3 \times 3$ as shown below:

\begin{tabular}{|c|c|c|}
\hline-1 & -2 & -1 \\
\hline 0 & 0 & 0 \\
\hline 1 & 2 & 1 \\
\hline
\end{tabular}

(a)

\begin{tabular}{|c|c|c|}
\hline-1 & 0 & 1 \\
\hline-2 & 0 & 2 \\
\hline-1 & 0 & 1 \\
\hline
\end{tabular}

(b)

Figure 2: Sobel 3-D Kernels

The Sobel operator uses differentiation operation for computing gradient in horizontal and vertical direction and then convolving the original image with the masks, edges in an image can be detected.

\section{Prewitt Operator}

The Prewitt Operator [3] is similar to Sobel operator. The only difference is more weight is attached with the central co-efficient in case of Sobel operator. The 2-D mask of Prewitt Operator is given as follows:

\begin{tabular}{|c|c|c|}
\hline-1 & 0 & 1 \\
\hline-1 & 0 & 1 \\
\hline-1 & 0 & 1 \\
\hline
\end{tabular}

(a)

\begin{tabular}{|c|c|c|}
\hline-1 & -1 & -1 \\
\hline 0 & 0 & 0 \\
\hline 1 & 1 & 1 \\
\hline
\end{tabular}

(b)

Figure 3: Prewitt 3-D Kernels

\section{Compass Operator}

Prewitt Operator and Sobel operator detects edges in vertical direction as well as in horizontal direction present in an image. But Compass Operator can detect edges in all directions present in an image as the mask rotates in 45 degree in the anticlockwise direction. A Prewitt or Sobel mask can be considered for this operation. It detects weak edges in an image efficiently.

\section{A. Laplacian of Gaussian}

\section{(2) Second order edge detection operators}

The Laplacian operator is an example of Second order edge detection operator. It gives very good performance. But this edge detection operator has two limitations mentioned below:

(1) It produces double edges for each edge i.e., one edge in the positive direction and another edge in negative direction.

(2) It is highly sensitive to noise.

These two limitations are removed by modifying the Laplacian operator as Laplacian of Gaussian operator which first performs smoothing operation by using a Gaussian low pass filter followed by Laplacian operator for detecting edges in an image.

The Laplacian of an image $\mathrm{f}(\mathrm{x}, \mathrm{y})$ can be expressed as

$$
\mathrm{L}(\mathrm{x}, \mathrm{y})=\frac{\delta^{\mathrm{n}} f}{\delta x^{2}}+\frac{\delta^{2} f}{\delta y^{2}}
$$

The Laplacian of Gaussian of an image $f(x, y)$ can be expressed as 


$$
\operatorname{LoG}(\mathrm{x}, \mathrm{y})=\frac{1}{\sqrt{2 \pi \sigma^{2}}} \exp \left(-\left(x^{2}+y^{2} / 2 \sigma^{2}\right)\right)
$$

This operator detects an edge in an image by taking the second derivative [4] [5] of image gradient and finding out the zero crossing. The performance of this operator depends upon the value of ' $\sigma$ ' where $\sigma$ is the standard deviation.

\section{B. Canny Edge Detector}

The Canny Edge Detector [6] is one of the most popular contemporary edge detection Operator. It performs edge linking \& connecting broken edge pixels by the following steps:

1. Apply the Gaussian low pass filter to the image

2. Compute the local gradient for each point in the smoothed image

3. Apply non maximal suppression to narrow the broad edges

4. Apply hysteresis thresholding to the image to detect edge pixels

\section{The Marr-Hildreth Edge Detector}

The Marr-Hildreth Edge Detector [7] is a very popular gradient based operator which uses Laplacian method to the second derivative of an image. The algorithm can be explained as follows:

(1) Apply the Gaussian low pass filter to smooth the input image

(2) Apply Mexican Hat operator[8] to find the second derivative of the image

(3) Set an appropriate threshold value to find the edge pixels(If the pixel value is greater than the threshold value, mark it as an edge)

\section{The Haralick Algorithm}

The Haralick Algorithm [9] is a second derivative based algorithm which can be used for detecting edges in an image. The input image is smoothed by local Bi-cubic polynomial fitting [10]

(1) Calculate the second derivative of the image

(2) Edges can be detected at the zero crossing points

\section{COMPARATIVE ANALYSIS}

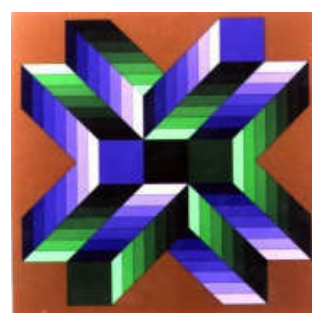

(a)

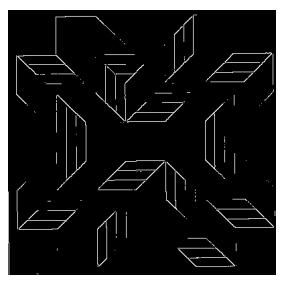

(c)

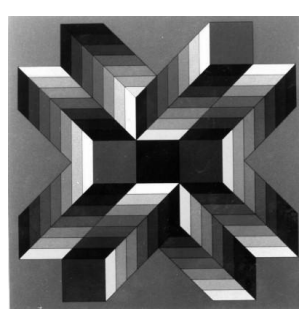

(b)

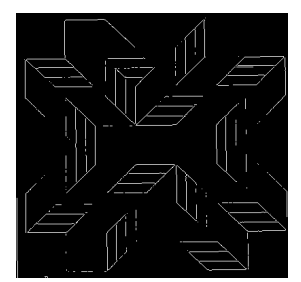

(d) 


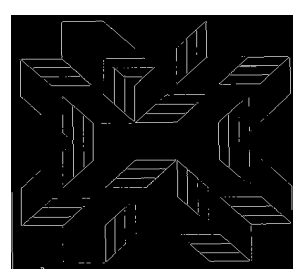

(e)

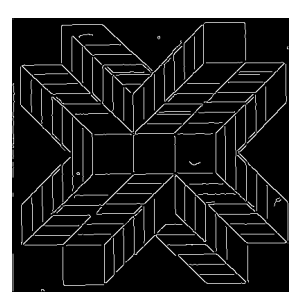

(g)

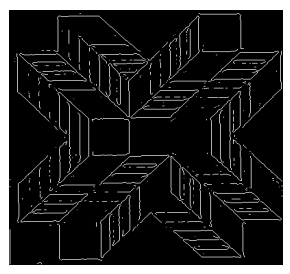

(f)

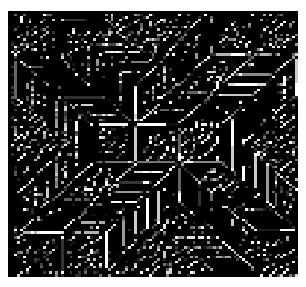

(h)

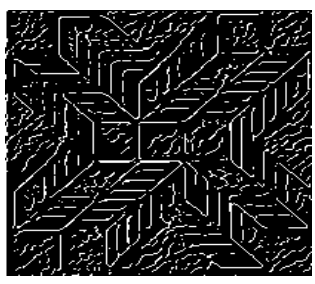

(i)

Figure 5: (a) Original color Image (b) Gray scale Image (c)Edge Detection with Robert's Operator (d) Edge Detection with Sobel's Operator (e) Edge Detection with Prewitt's Operator (f) Edge Detection with LoG Operator (g) ) Edge Detection with Canny's Operator (h) Edge Detection With Marr-Hildreth's Operator (i) Edge Detection With Haralick Operator

\section{IV.EXTENDING THE EDGE DETECTION TECHNIQUE TO COLOR IMAGES}

In case of color image, each pixel consists of three components (Red, Green and Blue).Therefore color edge detection [11] deals with vector valued techniques instead of scalar ones.

These techniques are further divided into two types:

(1) Monochromatic based techniques

(2) Vector valued Techniques

\section{(1) Monochromatic based techniques}

In case of monochromatic based techniques each color component is treated separately and in the end they combine together as a single image. The main drawback of this technique is the correlation of the color channels due to which 
some important information (such as edges having equal strength but in opposite direction) cannot be extracted from the image.

\section{(2) Vector valued Techniques}

In this case the color information are treated as color vectors and the difference between the norms of the color vectors are calculated.

\subsection{Color Variants of the Canny Operator}

Novak and Shafer suggest the extension of Canny operator [6] for edge detection. Kanade introduces this approach in [12].

The Canny edge detection consists of the following three steps:

1. Determine the partial derivative of the smoothed image

2. Find the magnitude and direction of the edge pixel

3. To eliminate broad edges, apply non-maximal suppression

\subsection{Cumani operator}

In 1989, Cumani [13] suggests a second order partial derivative method for detection of edges in color images. Cumani considered the color image as a 2-D vector field expressed as

$$
\mathrm{I}(\mathrm{x}, \mathrm{y})=\left\{\mathrm{I}_{\mathrm{R}}(\mathrm{x}, \mathrm{y}), \mathrm{I}_{\mathrm{G}}(\mathrm{x}, \mathrm{y}), \mathrm{I}_{\mathrm{B}}(\mathrm{x}, \mathrm{y})\right\}
$$

and suggested a new norm [13] of directional derivative which is quadratic in nature and known as squared local contrast. By calculating the extreme Eigen values [14], Eigen vectors and zero crossing points, the edges can be detected in an image.

\subsection{Vector Order Statistics Operators}

Earlier the order statistics [15] operators are proposed for morphological edge detectors [16] [17]. But later these operators are extended for color image filtering and edge detection as vector order statistics operators [18]. In case of vector order statistics operators, the color edge detectors are combined by the linear combination of sorted vector samples and the coefficients of linear combination results different edge detection. One of the examples of vector order statistic operators is vector median.

Color images can be considered as multichannel signals. [19].The Median filter used for noise removal in gray scale images can't be applied directly on color images because color image contains vector data. Therefore, vector distance measure can be used as a sub ordering principles for ordering the vector data. The vector pixels in a window are ordered on the basis of the sum of the distances between each vector pixel and other vector pixels in the window. The vector pixel having the smallest sum of distances is considered as the vector median. The rank of the vector median is one. The rank-conditioned median filter operates only on the corrupted pixels in which pixels in the filtering window are ranked according to their magnitudes and the central pixel is considered to be corrupted if it lies outside the trimming range [20, 21].Center-weighted median filter [22] shows better performance over the weighted median filter [23], as it emphasizes on the center pixel of the image.

\subsection{Difference Vector Operator}

Difference Vector Operator [24] is similar to first derivative operator. In this approach, each pixel is represented as a vector in RGB color space and the gradient can be calculated in four different directions $\left(0^{\circ}, 45^{\circ}, 90^{\circ}\right.$ and $\left.135^{\circ}\right)$ by convolving the pixel values with the kernels. The result can be compared with a suitable threshold value to detect edges in a color image.

\section{Other Methods}

On selection and combination of relevant color components for edge detection [25]: This paper presents a combination of color components for edge detection by selecting the most relevant components from each color space.

A morphological Gradient approach to color Edge detection [26]: In this paper a color edge detector based on vector differences has been proposed to provide the color gradients

A novel Edge detection algorithm for Digital Mammogram [27]: Here the proposed algorithm produces edge map image which contains single pixel continuous line and it is free from noises. It separates the ROI from the exterior parts clearly for further analysis.

Gabor Filter and Rough clustering based Edge Detection [28]: The proposed method is based on Gabor Filter and rough clustering. The Gabor Filter smoothen the image and the rough clustering focuses on detection of edges. But this method fails to eliminate the image shadow. 
Vector Order Statistics operator as color edge detector [29]: A method based on vector order statistics is proposed here in which the detectors detect the local minima and maxima in the image and combine them suitably to produce an edge pixel. The R-ordering (Reduced ordering) method is adopted and a class of color edge detectors is defined using linear combination of sorted vector samples.

A novel approach to edge detection in color images by Genetic Algorithm and Artificial Neural Network [30]: In this paper a new algorithm is proposed which consists of noising models, de-noising models, Genetic algorithm and Artificial Neural Networks. It gives better results compared to traditional algorithms.

Color Edge Detection in presence of Gaussian Noise using nonlinear Pre filtering [31]: The proposed method adopts a multi pass processing approach which reduces noise in $R, G$ and $B$ components of the image. The filter is the combination of non-linear models and the proposed algorithm has been designed in such a way that it reduces the error caused by Gaussian noise even after pre filtering.

An improved automatic isotropic color edge detection technique [32]: In this paper the edge detection process is classified into two types:

(a) Feature extraction for calculating edge strength

(b) Feature classification automatic edge detection

A new method known as automatic isotropic color edge detection technique is developed for solving the feature extraction problem.

Edge and Corner detection by Color Invariants [33]: This paper presents a method in which a color saturation invariant based on dichromatic reflection model is first constructed and then it is synthesized with existing hue values to detect edges and corners in an image.

Cubical Voxels and Virtual electric field model for edge detection in color images [34]: Here the proposed idea is to model the color image as a network of elementary cubic points (voxels) of virtual electronic charges. These charges are uniformly distributed on a cube in electrostatic balance. This method is based on the combination of simple cubical voxels and virtual electric field models. Both are used simultaneously to describe the color images including more edges than usually used.

Application of new advanced CNN structure with adaptive thresholds to color edge detection [35]:

This paper presents adaptive templates which detect various color and intensity changes in in color image.

Edge Flow: A technique for boundary detection and image segmentation [36]: A boundary detection method based on "edge flow" is proposed in this paper. This method utilizes a predictive coding model to identify the change in color and texture at each image location at a given scale and constructs an edge flow vector. By iteratively propagating the edge flow, the boundaries can be detected at image locations which encounter two opposite directions of flow in the stable state. A user defined image scale is the only significant control parameter that is needed by the algorithm. This framework utilizes a predictive coding model to identify and integrate the direction of change in various types of image attributes (color, texture and phase discontinuity) at each image location and constructs an edge flow vector which points to the closest image boundary. But it is not appropriate for images which contain multiple scale information and the scale parameters need to be controlled locally.

An improved method of color image edge detection based on one order gradient operator [37]: Here the color contrast enhancement algorithm is used. Then it is compared with the gray edge detection operator, the extraction of color edge is implemented. It provides more edge information without leakage phenomenon. But it has limitations like, more time requirement.

Edge detection in different color spaces [38]: In this paper edge detection is applied on YIQ \& HSV instead of RGB only and compares visual performances. The result shows HSV color space is the most appropriate color space amongst the three.

Improving and measuring color edge detection algorithm in RGB color space [39]: In this paper, an enhancement of Dutta \& Choudhari algorithm is proposed. A complexity analysis, execution time and number of basic operations is shown with comparison to Canny \& Sobel edge detectors. The limitation of this method is the proposed algorithm needs more execution time than Sobel.

Human face detection in cluttered color images using skin color and edge information [40]: In this paper a fast algorithm is proposed for detecting human faces in color images. The algorithm uses color histogram for skin (in HSV space) in conjunction with edge information to locate faces in an image quickly. The algorithm locates faces but doesn't give exact contour and false alarm occurs when hand comes into picture.

Color dipole moments for edge detection [41]: A new approach based on adapting two well-known physical quantities of electromagnetism to the image maps is proposed in this paper. The dipole and quadruple moments of charge distribution can be defined for gray scale images and used in edge detection. Each pixel is associated with a color change; each change is connected with one of the three primary colors and the local distribution of color 
changes are used to define the dipole. With the evaluation of moments on small neighborhoods of each pixel, it is possible to detect the edges in image frame.

Color edge detection in presence of Gaussian Noise using Nonlinear pre-filtering [42]: The proposed method adopts a multi pass processing i.e. multi pass pre-filtering approach that gradually reduces the noise in the R, G, B components of the image. The algorithm operates in the column vector space that has been specifically designed to reduce the error caused by Gaussian noise if affecting the data after pre-filtering. It gives more accurate result.

Color image edge detection using Quaternion Quantized localized phase [43]:

In this paper, a new method is proposed to obtain more edge and contour information of interested image from its quantized localized space rather than from its magnitude as the phase information is of greater importance on edge areas than on smooth areas. By applying 2-D QSTFT (two dimensional quaternion short term Fourier Transform) to color image, locally quantizing the phase part of quaternion polar form of the transferred image, the edge information can be preserved.

Variational Restoration and Edge Detection for Color Images [44]: The extensions of Mumford-Shah functional for Color images are analyzed in this paper .The concept of images as surface is the main motivation. In this paper, some possible generalizations of the Mumford-Shah functional to the Color images, based on a geometric model of images as manifolds are implemented. The method is comparatively slow.

Color edge detection using the minimal spanning tree [45]: In this paper, a new approach based on minimal spanning tree for ordering and clustering multivariate data is proposed. It has the ability of rank multivariate data, preserve hierarchy and facilitate clustering. The MST is used for the identification of the pixels color distribution and their assignment to the different modes.

Comparing color edge detection techniques [46]: In this paper, both monochromatic and vector valued color edge detection techniques are analyzed by using their gray level counterparts. For analysis, monochromatic Sobel, Laplacian of Gaussian (LoG) and Canny are used with vector valued color edge detectors.

Color edge detection hardware based on geometric algebra [47]: Rotor convolution operation was introduced in this paper .This convolution operation belongs to a class of linear vector filters which can be applied to image on speech signals where vector filtering is of fundamental interest.

A new image edge detection method using quality-based clustering [48]: The proposed method uses k-means clustering coupled with silhouette analysis which is a good method for detecting edge for noisy images. This method shows higher noise resistance compared to other.

Improved edge detection by the evaluation of color contrast information [49]: In this paper, a method based on an active vision system based on neural modes "NAVIS" is proposed. It has five functional areas: camera control, form detection, attractivity calculation, attention selection and object recognition.(NAVIS : Neural-Active-VisionSystem: designed to recognize complex objects within a non-uniform environment). The form module initially consisted of gray value channel for the computation of luminance contrast. Here the gray value channel is extended by two color opponent channels for extraction of edges. This model achieved a new estimation of edges caused by luminance and color contrast. The attention and recognition capabilities of NAVIS can be improved by this method.

Structured forests for fast edge detection [50]: In this paper, a generalized structured learning approach is applied for edge detection. This method is computationally efficient.

A comparative study on color edge detection [51]: This paper presents a comparative study on color edge detection by using different approaches like Sobel operator, the Laplace operator, the Mexican hat operator, different realization of the Cumani operator and the Alshatti- Lambert operator. Also it presents an efficient algorithm for implementing Cumani \& Alshatti- lambert operator. Improved result is shown by using Gaussian masks with larger standard deviations instead of $3 \times 3$ mask.

Edge detection analysis [52]: In this paper, various edge detection algorithms \& edge detector design methods have been discussed. Some of them are: $1^{\text {st }}$ order derivative edge detection, $2^{\text {nd }}$ order derivative edge detection, edge detection using edge filtering method, color edge detection, line and spot detection.

Color edge detection by photometric quasi invariants [53]: In this paper, a set of quasi invariant derivatives are proposed like classical invariants which are insensitive to certain photometric variations such as shadow/shading/specular edges. Results show quasi invariants are well suited for edge detection of poor illumination \& low quality images.

Edge detection, spatial smoothing and image reconstruction with partially observed multivariate data [54]: In this paper, incomplete multivariate spatial data are considered to find out the missing data in the presence of edge. The methods based on Bayesian hierarchical models are examined \& compared with another method that imputes the missing data using edge preserving spatial smoothness with locally varying weights. But recovering of the information from the multivariate data is difficult. 
Detection and classification of edges in color images [55]: This paper presents a review of vector- valued techniques for detection \& classification of edge in color images. It shows color edge detectors detect more edges than gray level edge operators.

Discriminative sparse image models for class specific edge detection \& image interpretation [56]: In this paper, a multiscale method is proposed to minimize least squares reconstruction constraints. Applied to detection, category based edge selection \& image classification tasks.

Advantage: Able to learn local image descriptions that effectively support demanding computer vision tasks.

Learning to detect natural image boundaries using local brightness, color \& texture cues [57]: This paper introduces the detection \& localization of boundaries in natural scenes using local image measurement like brightness, color \& texture cues. Result shows a proper treatment of texture is essential for detection boundaries in natural images.

Edge detection techniques: Evaluation and Comparison [58]: In this paper, various edge detection techniques are compared. Boolean edge detector performs same as canny edge detector even if they took different approaches. Canny's edge detector is still preferred as it produces single pixel thick, continues edge. Boolean edge detector's edges are spotty. Multi-flash edge detection is better compared to others except one case where edges between are at same depth.

Color edge detection with least square numerical method [59]: In this paper, least square approximation method is used for edge detection in color images. The algorithm depends on the local scan of the image in 4 different directions (horizontal, vertical, major diagonal \& diagonal direction) \& standard Sobel operators are used comparison of results.

Color edge detection with the compass operator [60]: Compass operator is used for edge detection which substitutes vector quantization \& the earth mover's distance for Gaussian smoothing \& taking derivatives. It is stable \& more powerful than Canny's edge detector. It can be applied to any zero-mean convolution mask.

Color edge detection using the Skellam distribution as a sensor noise model [61]: In this paper, a noble edge detector based on the noise distribution for CCD or CMOS cameras is proposed. Skellam parameters are estimated by means and variance of Skellam distribution in the homogeneous patches. Result shows the proposed method can defeat fine details of the scene structure \&reduces noise. The responses around the corners are preserved well compared to other edge detectors.

Face detection in digital images using color spaces \& edge detection techniques [62]: A new algorithm called "PRECAN ALGORITHM" is proposed in this paper which is a combination of Canny \& Prewitt edge detectors and a combination of color spaces are used to detect the skin pixels. Efficiency of the proposed method is $84 \%$.It could be better by reducing the false missing hit.

Comprehensive analysis of edge detection in color image processing [63]: This paper presents a comparative study of some edge detectors. Here vector order statistic operators \& difference vector operators studied in details. The result shows DV operators with adaptive pre-filtering are superior over other detectors

Efficient color boundary detection with color-oppponent mechanisms [64]: The proposed method introduces a new framework for boundary detection in complex natural scenes based on the color opponent mechanism of the visual system. It is a feed forward hierarchical model, which has direct counterpart to the color opponent mechanisms involved in from retina to primary visual cortex. Result shows excellent capability of detecting both color \& luminance boundaries synchronously in a time saving manner.

Edge Detection by Curve Fitting [65]: In this paper, edge detection is considered as a curve fitting problem. High gradient pixels are grouped into elongated regions and then a curve is fitted to each. Weighed averaging idea is used here to reduce the noise and determining the edges.

\section{ANALYSIS OF QUATERNION-BASED METHODS}

The concept of quaternion is useful for color image processing and recognition. It was first proposed by Hamilton in 1843[66].It is a four-dimensional vector space defined over real numbers. A quaternion [67] can be represented as

$$
q=q_{0}+q_{1} i+q_{2} j+q_{3} k
$$

Where $\mathrm{q}_{0}, \mathrm{q}_{1}, \mathrm{q}_{2}, \mathrm{q}_{3}$ denote real coefficients and $\{1, \mathrm{i}, \mathrm{j}, \mathrm{k}\}$ form the basis of quaternion vector space and the quaternion $\mathrm{q}$ is called pure quaternion when $\mathrm{q}_{0}$ is equal to zero. The pure quaternion can be used as a mathematical tool to process color pixels $(\mathrm{R}, \mathrm{G}$ and $\mathrm{B})$ as a single pixel.

The conjugate of $q$ can be defined as

$$
\mathrm{q}^{\mathrm{c}}=\mathrm{q}_{0}-\mathrm{q}_{1} \mathrm{i}-\mathrm{q}_{2} \mathrm{j}-\mathrm{q}_{3} \mathrm{k}
$$


Alternatively, $\mathrm{q}$ and $\mathrm{q}^{\mathrm{c}}$ defined in the above equations (8) and (9) can be represented by $\mathrm{q}=\left(\mathrm{q}_{0}, \mathrm{q}_{1}, \mathrm{q}_{2}, \mathrm{q}_{3}\right)$ and $\mathrm{q}^{\mathrm{c}}=($ $\left.\mathrm{q}_{0},-\mathrm{q}_{1},-\mathrm{q}_{2},-\mathrm{q}_{3}\right)$ respectively. Also $\mathrm{q}$ and $\mathrm{q}^{\mathrm{c}}$ has same norms defined as

$$
|\mathrm{q}|=\left(\mathrm{q}_{0}^{2}+\mathrm{q}_{1}^{2}+\mathrm{q}_{2}^{2}+\mathrm{q}_{3}^{2}\right)^{1 / 2}
$$

The product of any two quaternions $\mathrm{i}, \mathrm{j}, \mathrm{k}$ is defined by the following multiplication rules:

$$
\begin{aligned}
& \mathrm{i}^{2}=\mathrm{j}^{2}=\mathrm{k}^{2}=-1 \\
& \mathrm{ij}=-\mathrm{ji}=\mathrm{k} \\
& \mathrm{jk}=-\mathrm{kj}=\mathrm{i} \\
& \mathrm{ki}=-\mathrm{ik}=\mathrm{j}
\end{aligned}
$$

The edge of color image can be found out by rotating the 3D vector of quaternion [68] [69].The magnitude of pure quaternion gives a particular color.

Some efficient methods of edge detection in color images using quaternion are listed below:

Color image edge based on quaternion convolution [70]: In this paper, a new edge-detecting filter is proposed which uses the idea of convolving color image with a pair of quaternion masks or hyper complex masks. The filter converts smoothly varying colored regions to grey and produces color where edge pixels are present.

Color edge detection using multiscale quaternion convolution [71]: In this paper, a color edge detector based on quaternion convolution is presented and also direction of edge is calculated for modulus maximum suppression. Color-sensitive Edge Detection using Hyper complex Filters [72]: In this paper, a color-pair-sensitive edge detection technique is proposed to find edges between homogeneous regions of particular colors using a hyper complex filter.

Color Image Edge Detection Using Quaternion Quantized Localized Phase [73]: This paper presents a novel method of color edge detection using quaternion polar form 2D quaternion short-term Fourier transform. It helps in preserving the edge information in the color images.

\section{VI.CONCLUSION}

In this paper, various monochrome edge detection techniques and color edge detection techniques were analyzed and comparison between them was presented. Firstly, the differences between the first derivative based edge detectors and the second derivative edge detectors were presented. In the next section, the vector-valued techniques were analyzed with respect to the monochromatic and gray-level edge detectors. In the vector order statistics operators, some well-known filters used for noise removal from color images such as vector median filter, weighted median filter, center weighted vector median filter were discussed. In the next part of the article some recent techniques used for color edge detection were presented. In the last section of the survey article, quaternions are discussed with their mathematical properties. Some papers were presented which proposed quaternion based techniques for edge detection in color images. The advantage of developing quaternion based edge detectors is that it combines a color 3-tuple to a hypercomplex number which can be processed as a single channel rather than as three different channels. It reduces the complexity of the method up to a large extent.

\section{REFERENCE}

[1] L. G. Roberts, "Machine Perception of Three-Dimensional Solids" Optical and Electro-Optical Information Processing. Tippet. J. T. (ed.) MIT Press, Cambridge, Mass, 1965.

[2] I. E. Sobel, Camera Models and Machine Perception, Ph.D. Thesis, Electrical Engineering Department, Stanford University, Stanford, CA, 1970.

[3] J. M. Prewitt, "Object Enhancements and Extraction,” in Picture Processing and Psychopictorics, B. Lipkin and A. Rosenfeld (eds.), Academic Press, New York, NY, pp. 75-149,1970.

[4] H.Sponton, J.Cardelino “A Review of Classic Edge Detectors”, http://www.ipol.im/,ISSN:2105-1232,July 2013

[5] R. C. Gonzalez and R. E. Woods, Digital Image Processing (3rd Edition). Prentice Hall, $3^{\text {rd }}$ ed., August, 2007.

[6] J. F. Canny, .A computational approach to edge detection,. IEEE Trans.Pattern Anal. Machine Intell, vol. 8, no. 6, pp. 679-698, November, 1986.

[7] D. Marr and E. Hildreth, "Theory of Edge Detection," Proceedings of the Royal Society of London, B207, pp. 187-217, 1980. 
[8] A. Koschan, "A comparative study on color edge detection." In Proceedings of the 2nd Asian Conference on Computer Vision, vol. 3, pp. 574-578. 1995

[9] R. M. Haralick, Ligital step edges from zero-crossings of second directional derivatives," IEEE Trans. Pattern Analysis and Machine Intelligence, vol. 6, pp. 58-68, January 1984.

[10] P. Getreuer, Linear Methods for Image Interpolation." http://www.ipol.im/pub/algo/g_linear_methods_for_image_interpolation/, IPOL 2011. ISSN: 2105-1232, DOI:.11

[11] P. Trahanias and A.N. Venetsanopoulos, "Color edge detection using vector statistics, "IEEE Trans. on Image Processing, vol. 2, pp. 259264, April 1993.

[12] T.Kanade, "Image understanding research at CMU," in Proc. Image Understanding Workshop, vol.II, pp.32-40, 1987.

[13] A. Cumani, "Edge Detection in Multispectral Images," CVGIP: Graphical Models and Image Processing. Vol. 53, pp. 40-51, 1991.

[14] W. Alshatti and P. Lambert, "Using eigenvectors of a vector field for deriving a second directional derivative operator for color images," in Proc. 5th Int. Conf. CAIP'93, pp.149- 156, 1993.

[15] H. A. David, Order Statistics, Wiley, New York, 1980.

[16] R. J. Feechs and G. R. Arce, “Multidimensional morphologic edge detection," in Conf. Visual Proc. SPIE 845, 285-292, 1987.

Communication and Image Processing,

[17] J. S. J. Lee, R. M. Haralick and L. G. Shapiro, “Morphologic edge detection,’ IEEE J. Robot Automat. RA-3(2), 142-156 ,1987.

[18] A. Koschan and M. Abidi," Detection and classification of edges in color images”, Signal Processing Magazine, Special Issue on Color Image Processing,22: 67-73, 2005.

[19] K.M.Singh, P.K.Bora, “Adaptive Vector Median Filter for Removal of Impulse Noise from Color Images”, JOURNAL OF ELECTRICAL \& ELECTRONICS ENGINEERING, Vol: 4, No: 1, pp.1063-1072, 2004.

[20] R. C. Hardie, K. E. Barner, "Rank-conditioned rank selection filters for signal restoration" IEEE Trans. Image Processing, Vol: 2, No: 2, pp. 192-206, 1994.

[21] Alparone L., Baronti S., Carlà R.,, "Two-dimensional rank-conditioned median filter," IEEE Trans. on Circuits and Systems - II: Analog and Digital Signal Processing, Vol: 42, No: 2,1995.

[22] S.J. Ko, Y.H. Lee, “Center-weighted median filters and their applications to image enhancement,” IEEE Trans. Circuits and Syst., Vol: 38, pp. 984-993, 1991.

[23] D.R.K. Brownrigg, “The Weighted median filter,” Comm. ACM, Vol: 27, pp. 807-818, 1984.

[24] S.Y. Zhu, K. N. Plataniotis, and A. N. Venetsanopoulos, "Comprehensive analysis of edge detection in color image processing," Opt. Eng.,vol. 38, no. 4, pp. 612-625, April 1999.

[25] A. Belmamoun, M. E. Hassouni and A. Hammouch, "On selection and combination of relevant color components for edge detection", Conference on Electronics, Telecommunications and Computers - CETC 2013, pp. 764 - 771, 2014.

[26] Evans, Adrian N., and Xin U. Liu. "A morphological gradient approach to color edge detection." Image Processing, IEEE Transactions on 15 , no. 6, pp. 1454-1463, 2006.

[27] M. I. Kanta, S. Nag, and S. K. Bandyopadhyay. "A Novel Edge Detection Algorithm for Digital Mammogram" International Journal of Information and Communication Technology Research 2, no. 2, 2012.

[28]Adak, Chandranath. "Gabor filter and rough clustering based edge detection." In Human Computer Interactions (ICHCI), 2013 International Conference on, pp. 1-5, IEEE, 2013.

[29] Trahanias, E.Panos, and A. N. Venetsanopoulos. "Vector order statistics operators as color edge detectors." Systems, Man, and Cybernetics, Part B: Cybernetics, IEEE Transactions on 26, no. 1 pp.135-143, 1996.

[30] N. Kaur, Rishma, N. Dhillon, "A novel approach to edge detection in color images by Genetic Algorithm and Artificial Neural Network", International Journal of Advanced Research in Computer Engineering \& Technology (IJARCET), Volume .3 ,Issue 1, January 2014.

[31] F. Russo and A. Lazzari, "Color edge detection in presence of Gaussian noise using nonlinear prefiltering", Instrumentation and Measurement, IEEE Transactions on, 54(1), 352- 358, 2005.

[32] J.Fan, W. G.Aref, M. S. Hacid, \& A. K. Elmagarmid, “An improved automatic isotropic color edge detection technique”. Pattern Recognition Letters,22(13), 1419-1429, 2001.

[33] J.Chu, J. Miao, G. Zhang and L. Wang, "Edge and corner detection by color variants", Optics \& Laser Technology, 45, pp.756-762, 2013. 
[34] B. Bouda, L. Masmoudi, \& D. Aboutajdine, "CVVEFM: Cubical voxels and virtual electric field model for edge detection in color images", Signal Processing, 88(4), 905-915, 2008.

[35] S.Deng, Y. Tian, X. Hu, P. Wei and M.Qin, "Application of new advanced CNN structure with adaptive thresholds to color edge detection", Communications in Nonlinear $\quad$ Science and Numerical Simulation, 17(4), 1637-1648, 2012.

[36] W. Y. Ma \& B.S.Manjunath, "Edge Flow: A technique for boundary detection and image segmentation", Image Processing, IEEE Transactions, Volume.9, Issue.8, pp.1375 - 1388, August 2000.

[37] W Jianwei, "An improved method of color image edge detection based on one order gradient operator", International Journal of Hybrid Information Technology, Vol.6, No.5, pp.151-162, 2013.

[38] G wanggil Jeon, "Edge detection in different color spaces", Proceedings, The 1st International Conference on Advanced Computer and Information Technology, ASTL Vol. 22, pp. 113 - 116, 2013.

[39] M. D. Almadhoun, "Improving and measuring color edge detection algorithm in RGB color space", International Journal of Digital Information and Wireless communications (IJDIWC) Vol.3, issue (1), pp.19-24, 2013.

[40] K.Sandeep and A.N.Rajagopalan, "Human face detection in cluttered color images using skin color and edge information", In ICVGIP, 2002.

[41] A.Sparavigna, "Color dipole moments for edge detection." arXiv preprint arXiv: 0904.0962, (2009).

[42] F Russo and A Lazzani, "Color edge detection in presence of Gaussian Noise using Nonlinear prefiltering", IEEE transactions on Instrumentation and Measurement, Vol.54, no.1,pp.352- 358, February 2005

[43] S.C. Pei and Y. Z. Hsiao, "Color image edge detection using Quaternion Quantized localized phase", $18^{\text {th }}$ European Signal Processing Conference, pp.1766-1770, August 2010.

[44] A Brook, R Kimmel and N. A. Sochen, "Variational Restoration and Edge Detection for Color Images", Journal of Mathematical Imaging and Vision Vol.18, pp. 247-268, 2003.

[45] Ch.Theoharatos, G.Economou and S.Fotopoulos, "Color edge detection using the minimal spanning tree", Pattern Recognition, Vol.38 pp.603-606, September 2005.

[46] H.I. Turhan, G. Sahin and A. M. Erkmen, “Comparing color edge detection techniques”.

[47] B. Misra and P.Wilson, "Color edge detection hardware based on geometric algebra" $3^{\text {rd }}$ European Conference on Visual Media Production, pp.115-121, November 2006.

[48] K.L. Chung, W.J. yang \& W.M. Yan and C.C. Wang, "Demosaicing of color filter array captured images using gradient edge detection masks and adaptive heterogeneity- Projection” IEEE Transaction on Image Processing, Vol. 17, No. 12, pp. 2356- 2367, December 2008.

[49] B. Neupane, Z. Aung, and W.L. Woon, "A new image edge detection method using quality- based clustering."

[50] M. Bollmann , T. Hempel and B. Mertsching, "Improved Edge Detection by the Evaluation of Color Contrast Information"1996.

[51] P.Dollár, and C. L. Zitnick. "Structured forests for fast edge detection." In Computer Vision (ICCV), 2013 IEEE International Conference on, pp. 1841-1848. IEEE, 2013.

[52] A. Koschan, “A Comparative Study on Color Edge Detection”, Proceedings 2nd Asian Conference on Computer Vision ACCV'95, vol. 3, pp. 574-578, December 1995.

[53] T.H.H. Lee and T.R.Taipei, "Edge detection analysis", Graduate Institute of Communication Engineering, National Taiwan University, Taipei, Taiwan, ROC, 2007.

[54] J.V.Weijer, T.Gevers and J.M. Geusebroek, "Color Edge Detection by Photometric Quasi-invariants" IEEE Trans. Pattern Analysis and Machine Intelligence, vol. 27 (4), pp.1520-1526, April 2005.

[55] S.C. Dass and V. N. Nair, "Edge detection, spatial smoothing and image reconstruction with partially observed multivariate data" Journal of the American Statistical Association Vol. 98, Issue 461,pp.77-89,2003.

[56] A. Koschan, M. Abidi, "Detection and Classification of Edges in Color Images: A Review of Vector-valued Techniques," IEEE Signal Processing Magazine, pp. 64-73, January 2005.

[57] J Mairal, M Leordeanu, Francis Bach, Martial Hebert and Jean Ponce,"Discriminative sparse image models for class specific edge detection \& image interpretation", Computer Vision - ECCV 2008,Lecture Notes in Computer Science, Vol. 5304, pp. 43-56, 2008.

[58] D.R.Martin, C.C.Fowlkes and Malik, "Learning to detect natural image boundaries using local brightness, color \& texture cues" IEEE Transactions on Pattern Analysis And Machine Intelligence, Vol. 26, no. 1,pp.1-20, January 2004. 
[59] E.Nadernejad, S.Sharifzadeh, H.Hassanpur, "Edge detection techniques: Evaluation and Comparison" Applied Mathematical sciences, Vol. 2, no. 31, pp.1507-1520, 2008.

[60] M. A. Ruxon \& C. Tomasi, "Color edge detection with the compass operator", IEEEConference on Computer Vision and Pattern Recognition '99, Vol. 2, pp.160-166, June 1999.

[61] Y. Hwang, I. Kweon \& J.S.Kim, "Color edge detection using the Skellam distribution as a sensor noise model", SICE Annual Conference, pp.1972-1979, September 2007.

[62] R. Ajmera and N. Saxena, "Face detection in digital images using color spaces \& edge detection techniques", International Journal of Advanced Research in Computer Science and Software Engineering, Vol. 3, Issue 6,pp.718-725, June 2013.

[63] K.N.Plataniotis, "Comprehensive analysis of edge detection in color image processing”, Optical Engineering, Vol. 38, No. 4, pp.612-62, April 1999.

[64] K.Yang, S.Gao, C. Li \& Y. Li, "Efficient color boundary detection with color-opponent mechanisms", IEEE Conference on Computer Vision and Pattern Recognition, pp.2810- 2817, June 2013.

[65] A.Goshtasby and H.L. Shyu. "Edge detection by curve fitting. "Image and Vision Computing 13, no. 3, pp.169-177, 1995.

[66] C.E. Moxey, S.J.Sangwine, T.A. Ell, "Hypercomplex Correlation Techniques for Vector Images ", IEEE Transaction on Signal Processing, vol.no.51, no.7, pp.1941-1953,2003.

[67] Pei, Soo-Chang, and Yu-Zhe Hsiao. "Colour image edge detection using quaternion quantized localized phase." In Signal Processing Conference, 2010 18th European, pp. 1766-1770. IEEE, 2010.

[68] F.Lang, J.Zhou, B.Yan, E.song, F.Jhong, “Quaternion and color Image Edge Detection”, Computer science, Vol.34, no.11, pp.221-216, 2007.

[69]L.Hua, H.Feng, K.Yu, Y.Liu, L.Ding, and J.Gu, "Edge Detectionfor Color Medical Image Based on Quaternion and SOFM-NN,"Int.J.of Advancements in Computing Technology, Vol.5, No.8, pp. 870-878, 2013

[70] S.J.Sangwine, "Colour image edge detector based on quaternion convolution." Electronics Letters 34.10, pp. 969-971,1998

[71] Xu, Jiangyan, L Ye, and W Luo. "Color edge detection using multiscale quaternion convolution." International Journal of Imaging Systems and Technology 20.4, pp. 354-358, 2010.

[72] Evans, Carolyn J., S. J. Sangwine, and T. A. Ell. "Colour-sensitive edge detection using hypercomplex filters." Signal Processing Conference, 2000 10th European. IEEE, 2000.

[73] Pei, Soo-Chang, and Yu-Zhe Hsiao. "Colour image edge detection using quaternion quantized localized phase." Signal Processing Conference, 2010 18th European. IEEE, 2010. 